"Mircea cel Batran" Naval Academy Scientific Bulletin, Volume XX - 2017 - Issue 2

The journal is indexed in: PROQUEST I DOAJ / Crossref / EBSCOhost/ INDEX COPERNICUSI OAJI / DRJI I

JOURNAL INDEX I I2OR / SCIENCE LIBRARY INDEX / Google Scholar / Academic Keys I ROAD Open Access I Academic Resources / Scientific Indexing Services / SCIPIO/ JIFACTOR

\title{
EXTENDING THE RANGE OF STABILITY FOR THE PERIODIC ORBITS OF ONE- DIMENSIONAL MAPS
}

\author{
Dumitru DELEANU ${ }^{1}$ \\ ${ }^{1}$ Assoc. Professor, PhD. Eng., General Engineering Sciences Department, Constanta Maritime University \\ 104, Mircea cel Batran street, Constanta, Romania, e-mail adress: dumitrudeleanu@yahoo.com
}

\begin{abstract}
Some dynamical systems with biological significance are well described by mappings depending sensitively on one or more parameters. The environment fluctuations can change these parameters and, along with them, the desired behavior of the system. In order to maintain a fixed activity (even in such conditions), a number of control approaches have been proposed. In the paper, extensive computer simulations have been provided to show the efficacy of a simple method suggested by N.A. Magnitskii in locating and stabilizing the unstable periodic orbits (UPOS) of chaotic maps. A rule to generate the control parameters is proposed and its rightness is checked for the case of two one-dimensional maps, one describing the malignant tumor growth and the other the dynamics of a single-species population with nonoverlapping generations.
\end{abstract}

Keywords: Control method, Chaotic maps, Unstable periodic orbits.

\section{INTRODUCTION}

A large number of biological systems are wellmodelled by discrete-time nonlinear equations. Even in the one-dimensional version, such equations are able of displaying a rich spectrum of dynamical behaviors including fixed points, limit cycles, bi-stability or chaos [ $1-3]$. The difference between these different comportments is given by one or more variable parameters which, in a biological system, may be quantities as rate of birth or death of different populations, rate of increase of malignant tumors, carrying capacities, and so on. As a consequence of mutations in the environment, these parameters can change radically, departing well from the usual values. The immediate result consists in forcing the dynamical system to behave very differently from what is desired. Thus, for systems that have a sensitive behavior to changing parameters, it is of considerable interest to dispose and implement mechanisms of control so to maintain the desired state even when the system is affected by external perturbations.

A simple method to achieve this goal was proposed by Magnitskii. It is applicable for the stabilization of the unstable periodic orbits of multi-dimensional chaotic maps and has been exemplified on well-known logistic and Henon maps [4].

In the paper, we apply the method to two onedimensional maps having biological relevance and propose a rule to generate the necessary control parameters.

\section{SHORT DESCRIPTION OF THE METHOD}

Consider the one-dimensional nonlinear map

$x_{n+1}=f\left(x_{n}, p\right), x_{n} \in R, n \geq 1$ where $p \in R$ is a parameter.

Let $P=\left(\bar{x}_{1}(p), \bar{x}_{2}(p), \ldots, \bar{x}_{s}(p)\right)$ be a period $-s$ orbit of map (1), meaning

$\bar{x}_{2}=f\left(\bar{x}_{1}\right), \bar{x}_{3}=f\left(\bar{x}_{2}\right), \ldots, \bar{x}_{1}=f\left(\bar{x}_{s}\right)$

$P$ is a fixed point of map $f^{(s)}=\underbrace{f \circ f \circ \ldots \circ f}_{s \text { times }}$. For many maps there exist a critical value $\bar{p}_{c r}^{(s)}$ of the parameter such that the $s$ - cycle (2) is stable for $p<\bar{p}_{c r}^{(s)}$ and unstable otherwise. The map has another attractor (ordered or chaotic) for $p>\bar{p}_{c r}^{(s)}$. The algorithm described below will allow locating and stabilizing the $s$ - cycle (2) to the right of the critical value of $p$. It is based on the following result [4]:

Let $\bar{x}_{i}(p)$ be a point of the period - s orbit $P$ of map (1) and $\bar{p}_{c r}^{(s)}$ the critical value of the parameter $p$ such that

$v=\frac{\partial f^{(s)}}{\partial x}\left(\bar{x}_{i}\left(\bar{p}_{c r}^{(s)}\right), \bar{p}_{c r}^{(s)}\right)=\prod_{i=1}^{s} \frac{\partial f}{\partial x}\left(x_{i}\left(\bar{p}_{c r}^{(s)}\right), \bar{p}_{c r}^{(s)}\right) \neq 1$

Then, there exists $\bar{p}_{c r, 1}^{(s)}>\bar{p}_{c r}^{(s)}$ such that the point $\left(\bar{x}_{i}(p), p\right)$ is an asymptotically stable fixed point of the two-dimensional map

$\left\{\begin{array}{l}x_{n+1}=f^{(s)}\left(x_{n}, p\right)+\varepsilon\left(q_{n}-p\right) \\ q_{n+1}=f^{(s)}\left(x_{n}, p\right)-x_{n}+\beta\left(q_{n}-p\right)+p\end{array}\right.$ 
"Mircea cel Batran" Naval Academy Scientific Bulletin, Volume XX - 2017 - Issue 2

The journal is indexed in: PROQUEST / DOAJ / Crossref / EBSCOhost/ INDEX COPERNICUS/ OAJI / DRJI I JOURNAL INDEX / I2OR / SCIENCE LIBRARY INDEX / Google Scholar / Academic Keys / ROAD Open Access I Academic Resources / Scientific Indexing Services / SCIPIOI JIFACTOR

for every $p \in\left[\bar{p}_{c r}^{(s)}, \bar{p}_{c r, 1}^{(s)}\right\rfloor$, where $\beta=-v$ and $\varepsilon=\frac{v^{2}}{v-1}$

Remarks: i) The starting point for map (3) is $\left(\bar{x}_{i}\left(\bar{p}_{c r}^{(s)}, p\right)\right)$;

ii) The other points of the watched $s$ - cycle, $\bar{x}_{j}(p), j=\overline{1, s}, j \neq i$, are determined from (2);

iii) One can extend further the range of stability for the period orbit $P$. Thus, using $\bar{p}_{c r, 1}^{(s)}$ as the new critical value of $p$ and correcting the values for $\beta$ and $\varepsilon$ according to $v=\frac{\partial f^{(s)}}{\partial x}\left(\bar{x}_{i}\left(\bar{p}_{c r, 1}^{(s)}\right), \bar{p}_{c r, 1}^{(s)}\right)$, one can stabilize the point $\bar{x}_{i}(p)$ in another interval, $\quad p \in\left\lfloor\bar{p}_{c r, 1}^{(s)}, \bar{p}_{c r, 2}^{(s)}\right\rfloor$. This procedure is applicable as long as the orbit $P$ exists.

iv) For the particular maps discussed in the paper, there exist a simple rule to generate the required value $\beta_{k}$ for stabilizing the cycle (2) in the domain $p \in\left\lfloor\bar{p}_{c r, k}^{(s)}, \bar{p}_{c r, k+1}^{(s)}\right\rfloor$, namely

$$
\beta_{k+1}=\frac{2 \beta_{k}^{2}+2 \beta_{k}+1}{2 \beta_{k}+1}, k \geq 1, \beta_{0}=1
$$

The iterative formula (4) is proved in the Appendix. It follows that $\varepsilon_{k}=\frac{\beta_{k}^{2}}{1+\beta_{k}}$.

\section{NUMERICAL SIMULATIONS}

The algorithm described in the previous section was applied to different one-dimensional mappings. We report here the results obtained for two maps having biological significance. The first one model the malignant tumor growth and can be written as

$x_{n+1}=6.75 p\left(x_{n}^{2 / 3}-x_{n}\right) \equiv f\left(x_{n}, p\right)$

where the parameter $p \in[0,1]$ and the function $f$ transforms the interval $[0,1]$ onto itself [5].

The second map describes the dynamics of a single-species population which bread seasonally and have non-overlapping generations

$x_{n+1}=p x_{n}\left(1+\alpha x_{n}\right)^{-\delta} \equiv f\left(x_{n}, p\right)$

where $p$ denotes the finite rate of increase while $\alpha, \delta>0$ are parameters influencing the equilibrium density [6, 7].

Both maps possess a rich spectrum of dynamical behaviors, quite similar to logistic map, which includes fixed points, cascades of stable cycles and chaos. In what follows, we prove that scheme (3) is capable to locate and stabilize different periodic orbits of maps (5) and (6) far beyond the range of stability for the uncontrolled systems.

\subsection{Application of the control scheme (3) to map (5)}

The map (5) has two fixed points, namely $\bar{x}^{\prime}(p)=0 \quad$ and $\quad \bar{x}^{\prime \prime}(p)=\left(\frac{6.75 p}{1+6.75 p}\right)^{3}$. The second one is stable for $p \in(0.148,0.740)$ and then it is replaced, through a pitchfork bifurcation, by a period -2 orbit. This is, at its turn, stable for $p<\bar{p}_{c r}^{(2)}=0.898$ and then bifurcates into a 4cycle. The next bifurcations points are $\bar{p}_{c r}^{(4)}=0.924$ and $\bar{p}_{c r}^{(8)}=0.932$.
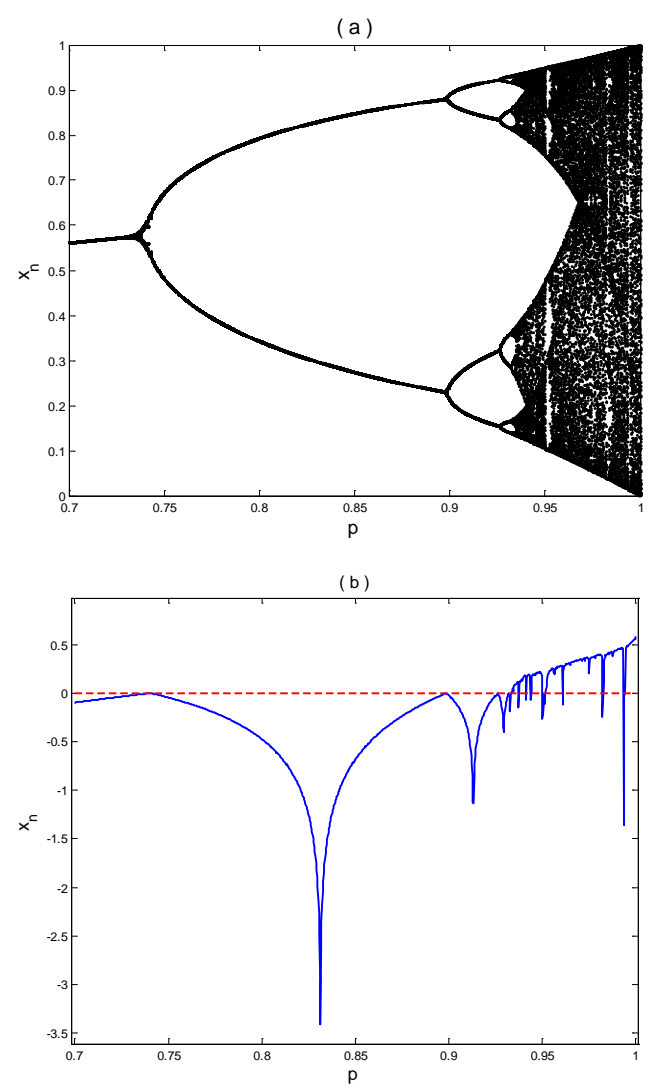

Figure 1. a) The bifurcation diagram for map (5);

b) The dependence of Lyapunov exponent on parameter $p$ for map (5).

Starting with an accumulation point, the map behaves chaotically, excepting some small windows with periodic behavior. Figure 1 shows the bifurcation diagram and Lyapunov exponent as a function of parameter $p \in[0.7,1]$.

We focus first on the nonzero fixed point noted, for simplicity, by $\bar{x}(p)$ and characterized by critical parameter value $\bar{p}_{c r}^{(1)}=0.74$. Because 
"Mircea cel Batran" Naval Academy Scientific Bulletin, Volume XX - 2017 - Issue 2

The journal is indexed in: PROQUEST I DOAJ / Crossref / EBSCOhost/ INDEX COPERNICUS/ OAJI / DRJI I JOURNAL INDEX / I2OR / SCIENCE LIBRARY INDEX / Google Scholar / Academic Keys / ROAD Open Access I Academic Resources / Scientific Indexing Services I SCIPIOI JIFACTOR

$\bar{x}\left(\bar{p}_{c r}^{(1)}\right)=0.5784$, one gets $v=-1$. The iterative scheme (3), with $\beta=1$ and $\varepsilon=0.5$, succeeded to locate properly and to stabilize the point $\bar{x}(p)$ on the entire interval $p \in[0.7,1]$. This was the output not only for the theoretical starting point $\bar{x}\left(\bar{p}_{c r}^{(1)}\right)$ but for almost all points $x_{1} \in[0,1]$. Figure $2 \mathrm{a}$ presents a typical evolution of the variables $x_{n}$ and $q_{n}$ towards the asymptotically stable point $(\bar{x}(p), p)$.
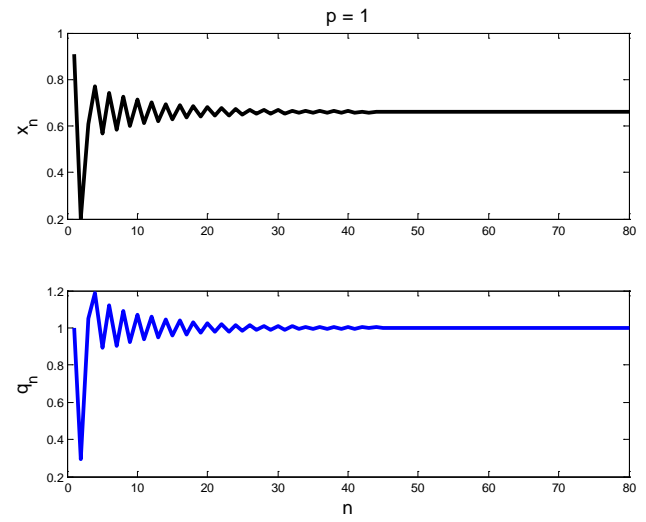

(a)
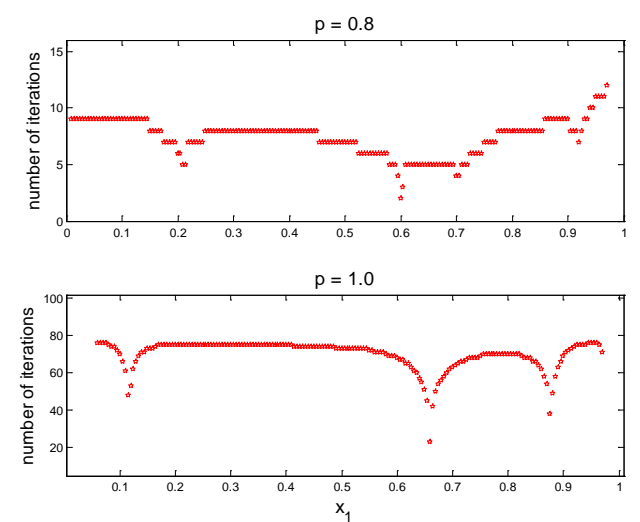

(b)

Figure 2. a) Stabilization of the fixed point of map (5) for $p=1$ and $\left.x_{1}=0.9 ; b\right)$ Dependence of the number of iterations until the map's (5) fixed point stabilization on the starting value $x_{1}$ for $p=0.8$ and $p=1.0$.

For a given $p$, the required number of iterations for stabilization does not vary significantly with the starting point, as illustrated in Figure $2 \mathrm{~b}$. This number is getting bigger once $p$ approaches 1 , but it remains inferior to the value 80 .

One can also locate and stabilize the periodic - 2 orbit on the interval $\left[\bar{p}_{c r}^{(2)}, 1\right]$ but this requires four steps. Using $\beta_{0}=1, \beta_{1}=\frac{5}{3}, \beta_{2}=\frac{89}{39} \quad$ and $\beta_{3}=3.7465$, we extended the range of stability for $p \in[0.898,0.935], p \in[0.935,0.967], p \in[0.967$
0.995], $p \in[0.995,1]$, respectively. One observes that the length of the interval $\left[\bar{p}_{c r, k-1}^{(2)}, \bar{p}_{c r, k}^{(2)}\right]$ becomes smaller and smaller as $k$ increases.

Figures 3 and 4 show the basins of attraction for the components $\bar{x}_{1}(p)$ and $\bar{x}_{2}(p)$ of the 2-cycle as well as the number of iterations until their stabilization for four representative values of parameter $p$. With the growth of $p$ we observe a marked erosion of the attraction basins so that close to $p=1$ only a few starting points $x_{1}$, close to the theoretical values, succeed to stabilize the 2-cycle's components.
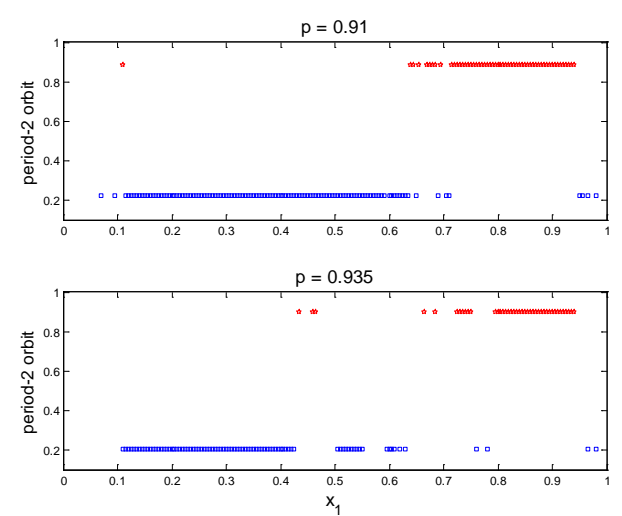

(a)
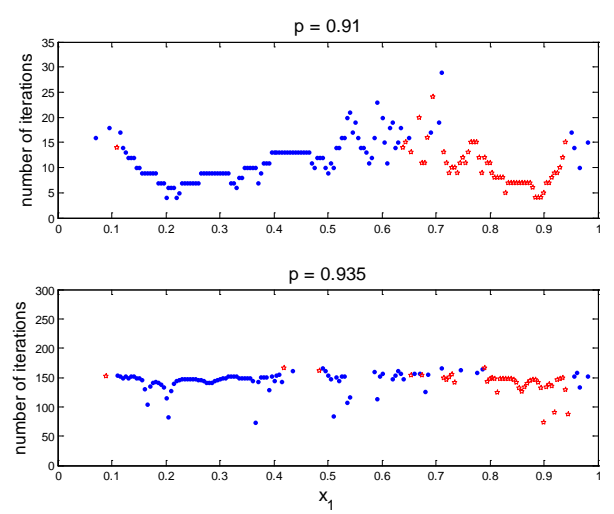

(b)

Figure 3. a) Basins of attraction for the map's (5) 2 cycle components when $p=0.91$ (up) and $p=0.935$ (down); b) Dependence of the number of iterations until the map's (5) 2-cycle stabilization on the starting value $x_{1}$ for $p=0.91$ (up) and $p=0.935$ (down). 
"Mircea cel Batran" Naval Academy Scientific Bulletin, Volume XX - 2017 - Issue 2

The journal is indexed in: PROQUEST I DOAJ / Crossref / EBSCOhost/ INDEX COPERNICUSI OAJI / DRJI I JOURNAL INDEX I I2OR / SCIENCE LIBRARY INDEX / Google Scholar / Academic Keys I ROAD Open Access I Academic Resources / Scientific Indexing Services / SCIPIOI JIFACTOR
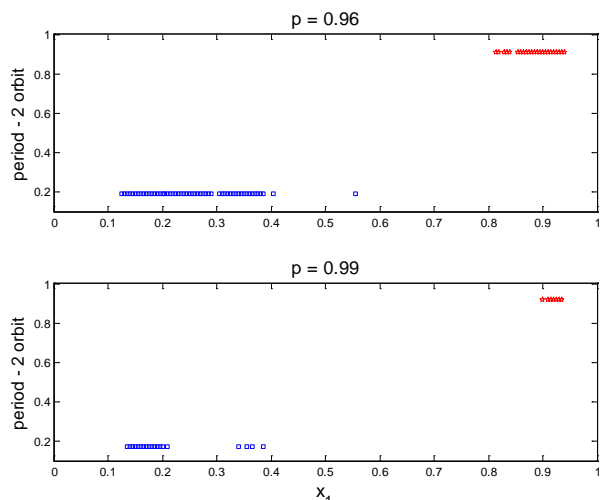

(a)
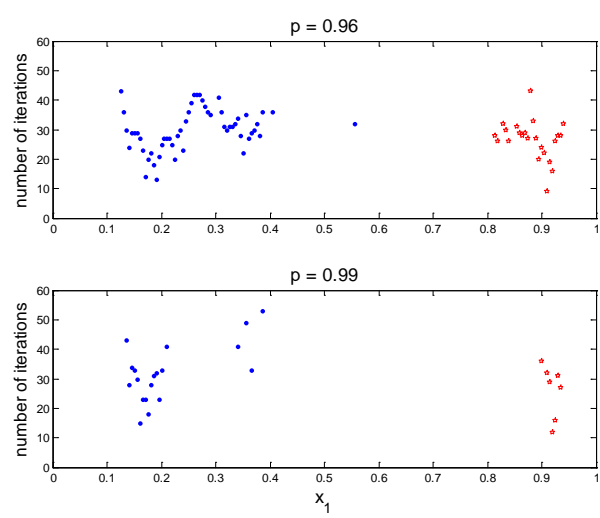

(b)

Figure 4. a) Basins of attraction for the map's (5) 2cycle components when $p=0.96$ (up) and $p=0.99$ (down); b) Dependence of the number of iterations until the map's (5) 2-cycle stabilization on the starting value

$$
x_{1} \text { for } p=0.96 \text { (up) and } p=0.99 \text { (down). }
$$

Lastly, we worked on stabilizing the period-4 orbit for $p>\bar{p}_{c r}^{(4)}=0.924$. Using the rule (4) to generate the control parameter $\beta$, we enlarged the range of stability for the 4-cycle up to $p=0.9715$ in seven steps, namely $p \leq 0.934$, $p \leq 0.9416, p \leq 0.9483, p \leq 0.9545, p \leq 0.9606$, $p \leq 0.9661$, and $p \leq 0.9483$. The lengths of these intervals decrease slowly and the process can continue by adding other stability sets. As with the period-2 orbit, the basins of attraction become increasingly narrow as $p$ is moving to higher values.

Figure 5 represents a graphical summary of our results so far. It contains the extensions for the stability regions of period-1, 2 and 4 orbits.

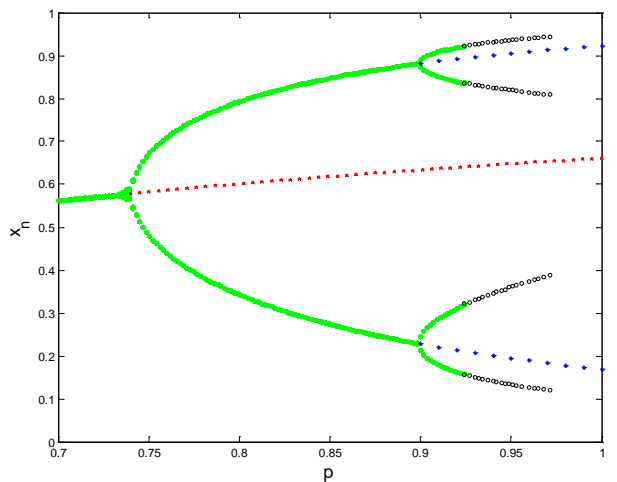

Figure 5. Extended regions of stability for period - 1, 2 and 4 orbits of map (5). Continuous (green) line denotes the intervals of stability for the uncontrolled map, while small (red and blue) stars and (black) circles stand for the controlled orbits.

3.2. Application of the control scheme (3) to map (6)

The non-trivial equilibrium is given by $\bar{x}(p)=\frac{p^{1 / \beta}-1}{\alpha}$ and it is stable for $\tilde{\beta}<2$, where $\tilde{\beta}=\beta\left(1-p^{-1 / \beta}\right)$, and unstable otherwise. If we consider the Nicholson's blowflies population (Zeirapthera fagi) the parameters' values are $\alpha=0.003$ and $\beta=6$, so $\bar{x}(p)$ becomes unstable starting with $\bar{p}_{c r}^{(1)}=\left(\frac{\beta}{\beta-2}\right)^{\beta}=11.3906$, when a period-2 orbit is born. The next bifurcations take place for $\bar{p}_{c r}^{(2)}=27.68$, $\bar{p}_{c r}^{(4)}=36.85$, and $\bar{p}_{c r}^{(8)}=39.41$. The bifurcation diagram for $p \in[0,80]$ is plotted in Figure 6, together with a magnification of it on $p \in[66,69]$, showing a regular behavior.

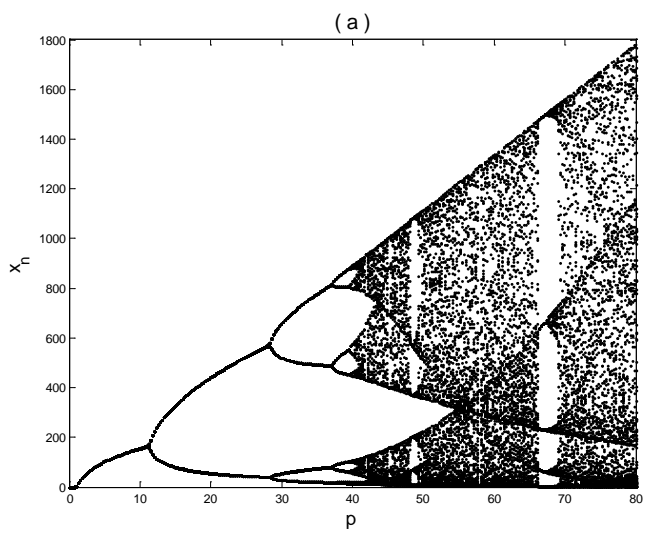


"Mircea cel Batran" Naval Academy Scientific Bulletin, Volume XX - 2017 - Issue 2

The journal is indexed in: PROQUEST I DOAJ / Crossref / EBSCOhost/ INDEX COPERNICUS/ OAJI / DRJI I JOURNAL INDEX / I2OR / SCIENCE LIBRARY INDEX / Google Scholar / Academic Keys / ROAD Open Access I Academic Resources / Scientific Indexing Services / SCIPIOI JIFACTOR

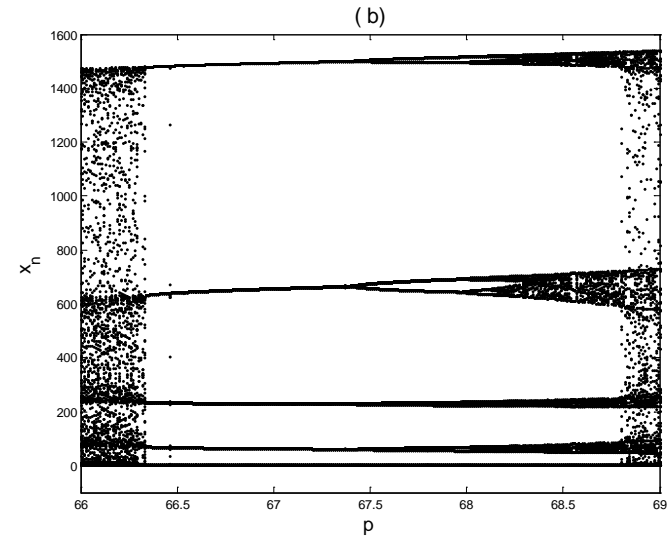

Figure 6. a) The bifurcation diagram of map (6) for parameter values $p \in[0,80]$; b) A magnification of the bifurcation diagram for $p \in[66,69]$.

The fixed point $\bar{x}(p)$ can be stabilized on the intervals $\left\lfloor\bar{p}_{c r}^{(1)}, 31.75\right\rfloor$ and $[31.75,80]$ with $\beta_{1}=1$ and $\beta_{2}=5 / 3$. Figure 7 reports the number of iterations until stabilization for $p=42$ and $p=80$. We observe the same trend as for map (5), that is a slowing down of the convergence process with increasing $p$. Additionally, the minimum effort for stabilizing the fixed point is required for initial condition $x_{1}$ close to $\bar{x}(42)=288.1368$ or $\bar{x}(80)=358.5939$, respectively.
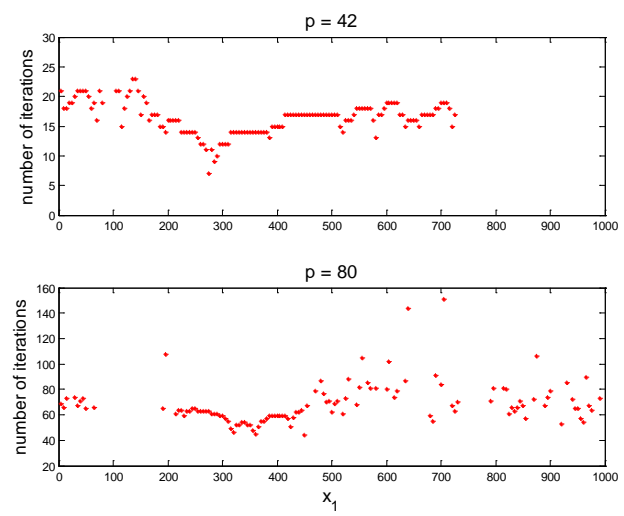

Figure 7. Dependence of the number of iterations until the map's (6) fixed point stabilization on the starting value $x_{1}$ for $p=42$ and $p=80$.

Concerning the period-2 orbit, it was stabilized on $\left.\mid \bar{p}_{c r}^{(2)}, 80\right\rfloor$ in three steps, $p \in[27.68,41.55]$, $p \in[41.5,65.58]$ and $p \in[41.58,80]$. As for the fixed point, we notice here an increase in length of consecutive intervals on which the 2-cycle is stabilized. Figure 8 shows, for different $p$, the number of iterations (and, implicitly, the basins of attraction) until the stabilization of the 2-cycle. Most of the initial conditions lead to one of the cycle components. Also, erosion of attraction basins with the increase of $p$ is easily noticeable.

DOI: 10.21279/1454-864X-17-12-020

(c) 2017. This work is licensed under the Creative Commons Attribution-Noncommercial-Share Alike 4.0 License.
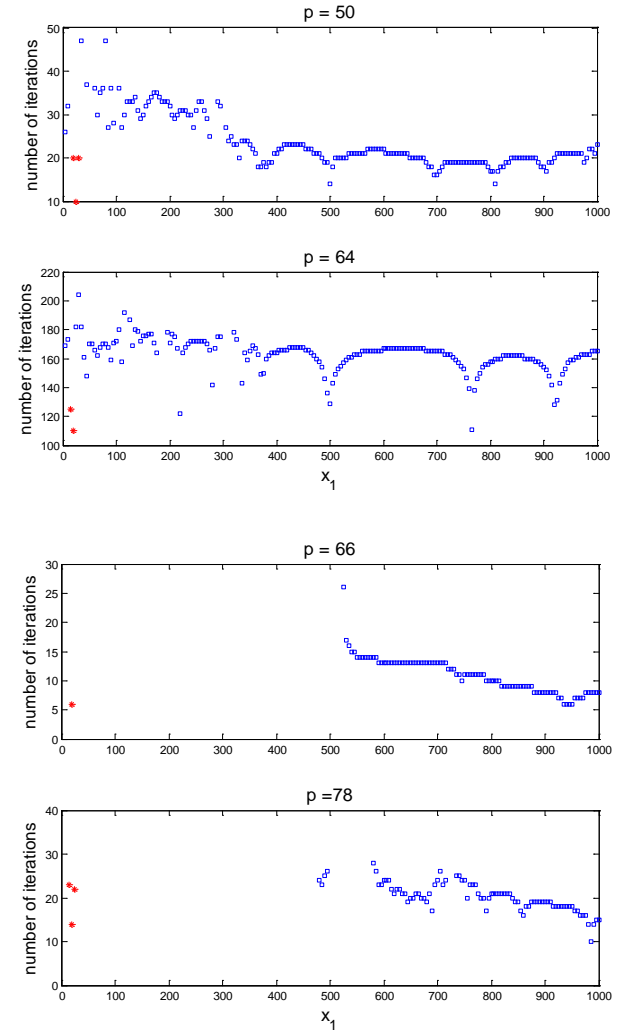

Figure 8. Dependence of the number of iterations until the map's (6) period-2 orbit stabilization on the starting value $x_{1}$ for different $p$.

The algorithm (3) allowed us to stabilize the period-4 orbit far to the right of the critical value $\bar{p}_{c r}^{(4)}$, but in much smaller steps than for the fixed point and period-2 orbit. The first five extensions have covered the intervals [36.85, 40.35], [40.35, 43.67], [43.67, 47.05], [47.05, 50.48] and [50.48, 54.05], having approximately equal lengths. Small changes to these ranges can be made by choosing different starting points. Figure 9 confirms the previous remarks on the reduction of attraction basins before the algorithm resumes with other control parameters and on the increased number of reiterations of the scheme (3) until a stable cycle is detected.

We close our numerical investigation with the period-5 orbit displayed in Figure $6 \mathrm{~b}$. It bifurcates into period-10 orbit for $p=67.425$ and into period20 orbit for $p=67.995$. Using $\beta_{1}=1$ and $\beta_{2}=5 / 3$ we extended its stability on [67.425, 68.259] and [68.259, 69.329]. The action can continue as long as this unstable orbit exists in the chaotic region. 
"Mircea cel Batran" Naval Academy Scientific Bulletin, Volume XX - 2017 - Issue 2

The journal is indexed in: PROQUEST / DOAJ / Crossref / EBSCOhost/ INDEX COPERNICUS/ OAJI / DRJI I JOURNAL INDEX / I2OR / SCIENCE LIBRARY INDEX / Google Scholar / Academic Keys / ROAD Open Access I Academic Resources / Scientific Indexing Services / SCIPIOI JIFACTOR
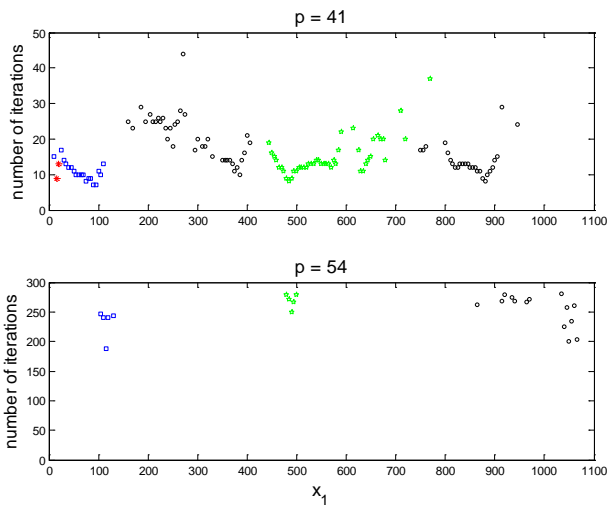

Figure 9. The number of iterations until the period-4 orbit of map (6) is stabilized. Up: $p=41 ;$ Down: $p=54$.

Different symbols (and colors) indicate the four component of the cycle.

\section{CONCLUSIONS}

In the paper, we have numerically analyzed a control method intended to localize and stabilize the UPOs of some discrete-time dynamical systems. The simulations were carried out on two one-dimensional maps having biological significance. The main conclusions of the study are as follows:

a) The method's algorithm allows to stabilize UPOs for parameter values much higher than a critical value, as long as the orbits exist;

b) The simple rule proposed in the paper for generating the control parameters worked extremely well. It permitted to extend the range of stability for the UPOs with low periods in a number of intervals, whose lengths are proportional to the size of stability domains;

c) For fixed control parameters, the basins of attraction for different components of an UPO became smaller and smaller as the system's parameter is increased, while the number of iterations until the stabilization goes up. When the basins vanish entirely, a new set of control parameters must be generated.

\section{APPENDIX}

The Jacobian matrix of map (3) at the point $\left(\bar{x}_{i}(p), p\right), p \in\left[\bar{p}_{c r, k-1}^{(s)}, \bar{p}_{c r, k}(s)\right]$, is

$J(p)=\left(\begin{array}{cc}\frac{\partial f^{(s)}}{\partial x}\left(\bar{x}_{i}(p), p\right) & \varepsilon_{k-1} \\ \frac{\partial f^{(s)}}{\partial x}\left(\bar{x}_{i}(p), p\right)-1 & \beta_{k-1}\end{array}\right)$

Its eigenvalues are provided by the characteristic equation

$\lambda^{2}-\left(D+\beta_{k-1}\right) \lambda+D \beta_{k-1}-\varepsilon_{k-1}(D-1)=0$

where $D=\frac{\partial f^{(s)}}{\partial x}\left(\bar{x}_{i}(p), p\right)$.

The point $\left(\bar{x}_{i}\left(\bar{p}_{c r, k-1}^{(s)}\right) \bar{p}_{c r, k-1}^{(s)}\right)$ is super-stable if both roots of (A2) are equal to zero. This implies

$D_{k-1}+\beta_{k-1}=0, D_{k-1} \beta_{k-1}-\varepsilon_{k-1}\left(D_{k-1}-1\right)=0 \Leftrightarrow$

$\beta_{k-1}=-D_{k-1}, \varepsilon_{k-1}=\frac{D_{k-1}^{2}}{1-D_{k-1}}=\frac{\beta_{k-1}^{2}}{1+\beta_{k-1}}$, with

$D_{k-1}=\frac{\partial f^{(s)}}{\partial x}\left(\bar{x}_{i}\left(\bar{p}_{c r, k-1}^{(s)}\right), \bar{p}_{c r, k-1}^{(s)}\right)$.

For a large set of chaotic maps, as $p$ increases the eigenvalues start to grow in magnitude and for $p=\bar{p}_{c r, k}^{(s)}$ one eigenvalue reaches the value -1 . Now, one has

$1+\left(D_{k}+\beta_{k-1}\right)+D_{k} \beta_{k-1}-\varepsilon_{k-1}\left(D_{k}-1\right)=0 \Leftrightarrow$ 
"Mircea cel Batran" Naval Academy Scientific Bulletin, Volume XX - 2017 - Issue 2

The journal is indexed in: PROQUEST / DOAJ / Crossref / EBSCOhost/ INDEX COPERNICUS/ OAJI / DRJI I JOURNAL INDEX / I2OR / SCIENCE LIBRARY INDEX / Google Scholar / Academic Keys / ROAD Open Access I Academic Resources / Scientific Indexing Services / SCIPIO/ JIFACTOR

$D_{k}=-\frac{2 \beta_{k-1}^{2}+2 \beta_{k-1}+1}{2 \beta_{k-1}+1}$ and, as a consequence

$\beta_{k}=-D_{k}=\frac{2 \beta_{k-1}^{2}+2 \beta_{k-1}+1}{2 \beta_{k-1}+1}, \varepsilon_{k}=\frac{\beta_{k}^{2}}{1+\beta_{k}}$

$D_{0}=\frac{\partial f^{(s)}}{\partial x}\left(\bar{x}_{i}\left(\bar{p}_{c r}^{(s)}\right), \bar{p}_{c r}^{(s)}\right)=-1, \beta_{0}=-D_{0}=1$.

\section{BIBLIOGRAPHY}

[1] R.M. May, Simple mathematical models with very complicated dynamics, Nature, 261, p. 459 - 467, 1976 [2] A.E. Elsadany, Dynamical complexities in a discrete-time food chain, Computational Biology and Software, 2 (2), p. $124-139,2012$.

[3] M.R.S. Raj, A.G.M. Selvam, M. Meganathan, Dynamics in a discrete prey-predator system, Int. Journal of Engineering Research and Development, 6 (5), p. 1 - 5, 2013.

[4] M.A. Magnitskii, Stabilization of unstable periodic orbits of chaotic maps, Computers Math. Appl., 34 (2 4), p. $369-372,1997$.

[5] S. Codreanu, M. Danca, Suppression of chaos in one-dimensional mappings, Journal of Biological Physics, 23, p. 1 - 9, 1997.

[6] D. Deleanu, V.F. Panaitescu, Controlling chaos in biological populations with non-overlapping populations, Recent Advances in Circuits, Telecommunications and Control (CCTC 2013), Paris, France, 2013.

[7] M.P. Hassell, Density-dependence in single-species populations, The Journal of Animal Ecology, 49, p. 283 - 295, 1975. 